\title{
Excitation of low frequency oscillations in a planetary magnetosheath by supersonic shear flow
}

\author{
N. Borisov ${ }^{1}$ and M. Fränz ${ }^{2}$ \\ ${ }^{1}$ Institute of Terrestrial Magnetism, Ionosphere and Radio Waves Propagation, (IZMIRAN), 142190 Troitsk, Moscow region, \\ Russia \\ ${ }^{2}$ Max-Planck-Institut für Sonnensystemforschung, 37191 Katlenburg-Lindau, Germany
}

Received: 26 October 2010 - Revised: 3 February 2011 - Accepted: 4 February 2011 - Published: 21 March 2011

\begin{abstract}
We show that the slow magnetosonic (SM) perturbations generated in the vicinity of the magnetopause, due to the excitation of the Kelvin-Helmholtz (K.-H.) instability in the case of a supersonic flow velocity, are transformed into fast magnetosonic (FM) waves which can propagate into the magnetosheath. Under the conditions discussed in this paper, the FM wave has negative energy in the stationary (magnetospheric) coordinate frame. Due to this the outgoing FM wave increases the growth rate of the K.-H. instability excited at the magnetopause. Within the linear theory, we investigate the influence of the excited FM wave on the growth rate of the K.-H. instability. Simultaneously we predict the transformation of the SM mode into kinetic Alfvén (KA) mode. Thus, in general, two types of waves with different polarizations (the KA wave and the FM wave) should appear in the magnetosheath due to the excitation of the K.-H. instability. At the same time, the SM perturbations are only present in the localized region where the K.-H. instability is excited. To correctly describe the excitation of waves, we use twofluid (for electrons and ions) magnetohydrodynamics. This approach is more general than the ideal magnetohydrodynamics and allows us to take into account the effects associated with the finite Larmor radius of ions. Also it can be used to investigate the K.-H. instability in a multi-component plasma, or in the case where the frequency of perturbations is of the order of the gyrofrequency of oxygen ions which may occur, for example, at the magnetosheath of Mars.
\end{abstract}

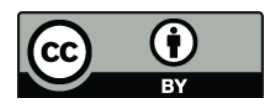

Correspondence to: $\mathrm{M}$. Fränz (fraenz@mps.mpg.de)

\section{Introduction}

One of the challenging problems in planetary physics is how the energy of the solar wind contributes to plasma processes inside the bow shock, in particular, in the magnetosphere. This problem is very important not only for the Earth, but also for the planets without global magnetic fields, like Mars and Venus. Experimentally it was established that for all these planets there is a boundary layer that separates the shocked solar wind plasma from the magnetospheric (ionospheric) plasma. This layer has a finite thickness across which the retardation (up to zero velocity) of the bulk plasma flow takes place.

The shear of the velocity can cause the K.-H. instability. This instability is widely known. It was investigated analytically and numerically in many publications (see, e.g., Chandrasekhar, 1961; Southwood, 1968; Ohsawa et al., 1976; Miura and Prichett, 1982; Pu and Kivelson, 1983; Choudhury and Lovelace, 1984; Miura, 1992; Fujita et al., 1996) discussing the generation of the LF perturbations at the magnetospheric boundary of Earth, Mars and Venus. For example, the K.-H. instability was called upon to explain the generation of the Pc-5 pulsations in the Earth's magnetosphere by surface oscillations at the magnetopause (Glassmeier, 1993; Sarafopoulos et al., 2001). Experimental data confirm that surface low-frequency oscillations at the Earth's magnetopause and Alfvén Pc-5 waves in the Earth's magnetosphere often appear simultaneously.

According to previous investigations in compressible plasma when the magnetopause is modelled by a tangential discontinuity there are two critical velocities for the K.-H. instability: the lower $v_{c, 1}$ and the upper one $v_{c, 2}$ (see, e.g. $\mathrm{Pu}$ and Kivelson, 1983). If the fluid velocity in the magnetosheath $V_{0}$ is smaller than $v_{c, 1}$ or higher than $v_{c, 2}$, there is no K.-H. instability at all. The existence of the upper critical

Published by Copernicus Publications on behalf of the European Geosciences Union and the American Geophysical Union. 
velocity $v_{c, 2}$ is often explained by the excitation of the fast FM wave when the flow velocity is high enough $V_{0}>v_{c, 2}$ (see, e.g. Mann et al., 1999). At the same time it is clear that when the FM wave is excited the boundary condition at the upper (magnetosheath) side should be changed. The boundary condition in the form of an attenuated wave becomes incorrect. It should be substituted by the condition corresponding to the wave leaking into the magnetosheath. Due to this the conclusion concerning the existence of the second critical velocity does not look very convincing. In 2-D simulations of the K.-H. instability, Lai and Lyu (2006) attracted attention to the excitation of the FM wave propagating into the magnetosheath for supersonic flow velocity. But the influence of such excitation on the growth rate of the K.-H. instability was not investigated.

In the papers which describe the flow interaction as a smooth layer of finite thickness, there is a statement that only one (the lower) critical value $v_{c 1}$ is present (see, e.g. Miura, 1992). In this paper, due to the applied boundary conditions, no perturbations exist in the magnetosheath beyond the upper boundary and it is quite natural that the instability exists no matter how large the flow velocity is (Choudhury and Lovelace, 1984; Miura, 1992).

While in the earlier papers, different aspects of the linear stage of the K.-H. instability were discussed, later papers focused on the nonlinear stage of this instability. Large scale dynamics were analysed numerically in 2-D and 3-D simulations based on MHD equations (see, e.g. Takagi et al., 2006; Fujimoto et al., 2006). These simulations have shown that for developed vortices a coupling with smaller (ion and electron) scales takes place. This in turn results in the penetration of solar wind plasma through the boundary layer (Fujimoto et al., 2006). The existence of the K.-H. vortices at the Earth's plasmapause was confirmed experimentally (see, e.g. Hasegawa et al., 2004b,a, 2006, 2009).

In our publication, we would like to attract attention to one consequence of the excitation of the K.-H. instability at the planets plasmapause that was not analysed before. While previously the investigations were concentrated on the influence of the K.-H. instability on the magnetospheric plasma, we discuss the excitation of waves in the magnetosheath due to the K.-H. instability. We will show that for supersonic plasma flow in the magnetosheath the K.-H. instability at the magnetopause results in excitation of FM waves that under some conditions propagate into the magnetosheath. In such a case, the growth rate of the instability changes significantly due to the negative energy of the FM wave in the magnetospheric reference frame. The outgoing FM wave causes an increase of the growth rate. The physics behind this is that the leakage of FM wave with negative energy from the region where the K.-H. instability is excited causes the increase of energy in the system. The manifestation of these results depends on several parameters, namely the flow velocity $V_{0}$, the plasma pressure, the width of the layer, where the velocity changes, etc. Note, that the role of waves with negative energy in relation with the K.-H. instability was mentioned in several publications (see, e.g. Mann et al., 1999; Taroyan and Erdélyi, 2002) but the influence of the outgoing FM wave with negative energy on the growth rate of the K.-H. instability of the SM waves was not discussed.

Our approach is based on two-fluid magnetohydrodynamics (for electron and ions) which can be easily generalized for a multi-component plasma (e.g., two sorts of ions - oxygen and hydrogen). Previously a two-fluid approach with the aim to discuss the K.-H. instability was used by Dobrowolny $(1972,1977)$ in the local approximation. In such an approximation the frequency and the growth rate of perturbations change with coordinates and, thus, does not provide the correct description of the instability.

We would like to mention that, with slight modification, our approach can also be used to describe the peculiarities of the instability and the wave propagation for the case when the frequency is of the order of the Larmor frequency of oxygen ions. For a better understanding of the physical mechanism, it is reasonable to start with an analytical approach. For this purpose the most simple 2-D geometry with straight magnetic field lines is used in this paper.

\section{Basic equations}

We introduce a system of coordinates in which the plasma moves along the magnetopause (z-axis) with velocity $v_{\mathrm{p}}$. Parameters of the medium (plasma density, electron and ion temperatures, magnetic field, flow velocity), in a general case, slowly vary along the $\mathrm{x}$-axis (across the magnetopause) and the medium is homogeneous along the y-axis. The layer in which the velocity $v_{\mathrm{p}}$ is changing has a finite thickness $2 d$. In the magnetosphere $x<-d$ this velocity is equal to zero. In the magnetosheath $x>d$ the velocity $v_{\mathrm{p}}$ reaches a maximum value $V_{0}$ and is constant for $x>d$. The magnetic field lines $\boldsymbol{B}_{0}$ are assumed to be parallel to the flow velocity vector. This situation is typical for the nightside magnetopause. A more general case $(\theta \neq 0)$ will be discussed in another publication.

To analyse the excitation of the K.-H. instability in the linear approximation, we use the continuity equations for electrons and ions, the equations of their motion and the Maxwell equations. The continuity equations take the form

$\frac{\partial n_{\alpha}}{\partial t}+\nabla \cdot\left(N_{0} \mathbf{v}_{\alpha}\right)+\nabla n_{\alpha} \mathbf{v}_{\mathrm{p}}=0$

Here $N_{0}$ is a non-disturbed plasma density, $n_{\mathrm{e}}, n_{\mathrm{i}}$ are the perturbations of electron and ion densities, $\boldsymbol{v}_{\alpha}$ are the velocities of perturbations for electrons and ions $\alpha=e, i$. The dependence of all perturbations on coordinates and time in general is expressed in the form $\propto f(x) \exp \left[i\left(\omega t-\int^{z} k_{\mathrm{z}} d z-k_{\mathrm{y}} y\right)\right]$. To take into account the Doppler shift $k_{\mathrm{z}} v_{\mathrm{p}}(x)$ we also introduce another frequency $\omega_{\mathrm{d}}=\omega-k_{\mathrm{z}} v_{\mathrm{p}}(x)$. Later on in this paper, we concentrate on the case when the perturbations 
along the $\mathrm{y}$-axis are absent $k_{\mathrm{y}}=0$. It is convenient to express the electric $\boldsymbol{E}$ and magnetic $\boldsymbol{b}$ fields as functions of scalar $\phi$ and vector $\boldsymbol{A}$ potentials:

$$
\begin{array}{r}
\boldsymbol{E}=-\nabla \phi-\frac{i \omega}{c} \boldsymbol{A} \\
\boldsymbol{b}=\nabla \times \boldsymbol{A}
\end{array}
$$

The linearized equation of motion for electrons can then be written as

$$
\begin{aligned}
i \omega_{\mathrm{d}} \mathbf{v}_{\mathrm{e}}+\left(v_{\mathrm{e}, \mathrm{x}} \frac{\partial}{\partial x}\right) \mathbf{v}_{\mathrm{p}}= & -\frac{\boldsymbol{e}}{m} \boldsymbol{E}-\frac{\nabla p_{\mathrm{e}}}{N_{0} m}-\omega_{\mathrm{He}}\left[\boldsymbol{v}_{\mathrm{e}} \times \boldsymbol{e}_{\mathrm{z}}\right] \\
& -\omega_{\mathrm{He}}\left[\boldsymbol{v}_{\mathrm{p}} \times \frac{\boldsymbol{b}}{\boldsymbol{B}_{0}}\right]
\end{aligned}
$$

Here $\boldsymbol{v}_{\mathrm{e}}$ is the perturbation of the fluid velocity of electrons, $m, e$ are the mass and the electric charge of electrons, $p_{\mathrm{e}}=\gamma T_{\mathrm{e}} \cdot n_{\mathrm{e}}$ is the perturbed pressure, $T_{\mathrm{e}}$ is the background temperature of electrons, $\omega_{\mathrm{He}}$ is the gyrofrequency of electrons, $\gamma$ is the adiabatic constant, $\boldsymbol{e}_{\mathrm{Z}}$ is a unit vector along the $\mathrm{z}$-axis (along the flow velocity). The equations of motion for ions

$$
\begin{aligned}
i \omega_{\mathrm{d}} \boldsymbol{v}_{\mathrm{i}}+\left(v_{\mathrm{i}, \mathrm{x}} \frac{\partial}{\partial x}\right) \boldsymbol{v}_{\mathrm{p}}= & \frac{e}{M} \boldsymbol{E}-\frac{\nabla p_{\mathrm{i}}}{N_{0} M}+\omega_{H_{\mathrm{i}}}\left[\boldsymbol{v}_{\mathrm{i}} \times \boldsymbol{e}_{\mathrm{z}}\right] \\
& +\omega_{\mathrm{Hi}}\left[\mathbf{v}_{\mathrm{p}} \times \frac{\boldsymbol{b}}{B_{0}}\right]-\frac{1}{M N_{0}} \nabla\{\pi\}
\end{aligned}
$$

contain the tensor of collisionless viscosity $\{\pi\}$ that should be taken into account to present correctly the kinetic Alfvén (KA) wave, $M$ is the mass of the ion, $\omega_{\mathrm{Hi}}$ is the gyrofrequency of the ion. The values for the components of the tensor $\{\pi\}$ can be found in Mikhailovskii and Onishchenko (1995).

From the Maxwell equation for the perturbed magnetic field it follows

$\nabla \cdot(\nabla \cdot \boldsymbol{A})-\Delta \boldsymbol{A}=\frac{4 \pi}{c} e N_{0}\left(\mathbf{v}_{\mathrm{i}}-\boldsymbol{v}_{\mathrm{e}}\right)$

The transverse and the longitudinal velocities of electrons expressed in terms of the electric potentials in the lowest approximation with respect to $\omega_{\mathrm{d}} / \omega_{\mathrm{He}}$ take the form

$$
\begin{aligned}
& v_{\mathrm{e}, \mathrm{x}}=-i \frac{e \omega_{\mathrm{d}}}{m c \omega_{\mathrm{He}}} A_{\mathrm{y}} \\
& v_{\mathrm{e}, \mathrm{y}}=+i \frac{e \omega_{\mathrm{d}}}{m \omega_{\mathrm{He}} c} A_{\mathrm{x}}+\frac{e}{m \omega_{\mathrm{He}}} \frac{\partial \phi}{\partial x}-\frac{\gamma}{m N_{0} \omega_{\mathrm{He}}} \frac{\partial T_{\mathrm{e}} n}{\partial x} \\
& v_{\mathrm{e}, \mathrm{z}}=-i \frac{e}{\omega_{\mathrm{d}} m} \frac{\partial \phi}{\partial z}+i \frac{\gamma}{N_{0} \omega_{\mathrm{d}} m} \frac{\partial T_{\mathrm{e}} n_{\mathrm{e}}}{\partial z}+i \frac{v_{\mathrm{e}, \mathrm{x}}}{\omega_{\mathrm{d}} \omega_{\mathrm{He}}} A_{\mathrm{y}} \frac{\partial v_{\mathrm{p}}}{\partial x}
\end{aligned}
$$

In the equations for ions, we have retained in the denominator the frequency $\omega_{\mathrm{d}}$. This is important for the excitation of the Alfvén waves with frequencies $\omega_{\mathrm{d}} \leq \omega_{\mathrm{Hi}}$.

$$
\begin{aligned}
v_{\mathrm{i}, \mathrm{x}}= & -i \frac{\omega_{\mathrm{Hi}}}{\omega_{\mathrm{Hi}}^{2}-\omega_{\mathrm{d}}^{2}} \frac{e \omega_{\mathrm{d}}}{M c} A_{\mathrm{y}} \\
& -i \frac{\omega_{\mathrm{d}}}{\omega_{\mathrm{Hi}}^{2}-\omega_{\mathrm{d}}^{2}}\left(i \frac{e \omega_{\mathrm{d}}}{M c} A_{\mathrm{x}}+\frac{e}{M} \frac{\partial \phi}{\partial x}+\frac{\gamma}{M N_{0}} \frac{\partial T_{\mathrm{i}} n_{\mathrm{i}}}{\partial x}\right)
\end{aligned}
$$

$$
\begin{aligned}
v_{\mathrm{i}, \mathrm{y}}= & \frac{\omega_{\mathrm{Hi}}}{\omega_{\mathrm{Hi}}^{2}-\omega_{\mathrm{d}}^{2}}\left(i \frac{e \omega_{\mathrm{d}}}{M c} A_{\mathrm{x}}+\frac{e}{M} \frac{\partial \phi}{\partial x}+\frac{\gamma}{M N_{0}} \frac{\partial T_{\mathrm{i}} n_{\mathrm{i}}}{\partial x}\right) \\
& +\frac{1}{\omega_{\mathrm{Hi}}^{2}-\omega_{\mathrm{d}}^{2}} \frac{e \omega_{\mathrm{d}}^{2}}{M c} A_{\mathrm{y}} \\
v_{\mathrm{i}, \mathrm{z}}= & -i \frac{e}{\omega_{\mathrm{d}} M} \frac{\partial \phi}{\partial z}+i \frac{\gamma}{\omega_{\mathrm{d}} M N_{0}} \frac{\partial T_{\mathrm{i}} n_{\mathrm{i}}}{\partial z}+i \frac{v_{\mathrm{i}, \mathrm{x}}}{\omega_{\mathrm{d}} \omega_{\mathrm{Hi}}} A_{\mathrm{y}} \frac{\partial v_{\mathrm{p}}}{\partial x}
\end{aligned}
$$

The terms with the collisionless viscosity are omitted in Eq. (7). However, they will be taken into account later to obtain the correct expression for the KA wave The velocities (6), (7) should be substituted into the Maxwell Eq. (5) and the continuity equations. The resulting system of equations, together with the equation of quasi-neutrality $n_{\mathrm{e}}=n_{\mathrm{i}}$, allow us to discuss the formation of the LF magnetosonic perturbations at the magnetopause.

\section{LF plasma perturbations at the magnetopause}

In this section, we neglect the effects associated with the finite Larmor radius of ions. Due to this we omit the tensor $\{\pi\}$ in the equation of motion for ions. It is assumed that small monochromatic perturbations exist along the zaxis $\propto \exp \left[i\left(\omega t-\int^{z} k_{\mathrm{z}} d z\right)\right]$.

Below, we consider the case when the frequency $\omega_{\mathrm{d}}$ at the magnetopause and in the magnetosheath is much smaller than the gyrofrequency of oxygen ions $\omega_{\mathrm{d}}^{2} \ll \omega_{\mathrm{Hi}}^{2}$. This case is usually discussed in the literature. We would like to derive a differential equation describing LF magnetosonic perturbations in the presence of a nonhomogeneous flow velocity of plasma $v_{\mathrm{p}}(x)$.

After the substitution of the velocities for electrons and ions (6), (7) into the Maxwell equation for the $A_{\mathrm{y}}$ component of the vector potential we find

$$
\left(\frac{\partial^{2}}{\partial x^{2}}-k_{\mathrm{z}}^{2}+\frac{\omega_{\mathrm{d}}^{2}}{c_{\mathrm{A}}^{2}}\right) A_{\mathrm{y}}=-\frac{4 \pi e}{c \omega_{\mathrm{Hi}}} \frac{\partial v_{\mathrm{s}}^{2} n}{\partial x}
$$

Here $c_{\mathrm{A}}$ is the Alfvén velocity $c_{\mathrm{A}}=\sqrt{\frac{B_{0}^{2}}{4 \pi M N_{0}}}, v_{\mathrm{S}}$ is the sound speed $v_{\mathrm{S}}=\sqrt{\gamma \frac{T_{\mathrm{i}}+T_{\mathrm{e}}}{M}}$.

The substitution of the velocities (6)-(7) into the continuity equations and elimination of the longitudinal electric field gives the following result

$$
\left(\omega_{\mathrm{d}}^{2}-k_{\mathrm{z}}^{2} v_{\mathrm{s}}^{2}\right) n=\frac{e \omega_{\mathrm{d}}^{2}}{c M} \frac{\partial}{\partial x}\left(\frac{N_{0}}{\omega_{\mathrm{Hi}}} A_{\mathrm{y}}\right)
$$

From the system of Eqs. (8)-(9) we find the equation for the potential $A_{\mathrm{y}}$

$$
\begin{aligned}
& \left(\frac{\partial^{2}}{\partial x^{2}}-k_{\mathrm{z}}^{2}+\frac{\omega_{\mathrm{d}}^{2}}{c_{\mathrm{A}}^{2}}\right) A_{\mathrm{y}}+\frac{4 \pi e^{2}}{M \omega_{\mathrm{Hi}}} \frac{1}{c^{2}} \frac{\partial}{\partial x} \frac{v_{\mathrm{S}}^{2} \omega_{\mathrm{d}}^{2}}{\omega_{\mathrm{d}}^{2}-k_{\mathrm{z}}^{2} v_{\mathrm{S}}^{2}} \frac{\partial}{\partial x}\left(\frac{N_{0}}{\omega_{\mathrm{Hi}}} A_{\mathrm{y}}\right) \\
& \quad=0
\end{aligned}
$$


The characteristic scales along the $\mathrm{x}$-axis of the plasma density $L_{N}=\frac{\partial N_{0}}{N_{0} \partial x}$, the magnetic field $L_{B}=\frac{\partial B_{0}}{B_{0} \partial x}$ and the fluid velocity $L_{\mathrm{V}}=\frac{2 d c_{\mathrm{A}}}{V_{0}}$ should be larger than the Larmor radius of ions $\rho_{\mathrm{Hi}}$ to use magnetohydrodynamics. Also, we suppose that quadratic combinations of the inverse scales are small enough $\left(L_{\mathrm{i}} L_{j}\right)^{-1} \ll k_{\mathrm{z}}^{2}$ and that the scale of plasma concentration is the largest $L_{B} / L_{N} \ll 1, L_{v} / L_{N} \ll 1$. In such an approximation, we arrive at the following equation

$$
\frac{\partial}{\partial x} \frac{\omega_{\mathrm{d}}^{2}\left(v_{\mathrm{S}}^{2}+c_{\mathrm{A}}^{2}\right)-k_{\mathrm{z}}^{2} v_{\mathrm{S}}^{2} c_{\mathrm{A}}^{2}}{c_{\mathrm{A}}^{2}\left(\omega_{\mathrm{d}}^{2}-k_{\mathrm{z}}^{2} v_{\mathrm{s}}^{2}\right)} \frac{\partial A_{\mathrm{y}}}{\partial x}+\left(\frac{\omega_{\mathrm{d}}^{2}}{c_{\mathrm{A}}^{2}}-k_{\mathrm{z}}^{2}\right) A_{\mathrm{y}}=0
$$

This equation describes two modes - the slow and the fast magnetosonic waves in a weakly inhomogeneous plasma. In the geometric optics approximation Eq. (11) reduces to

$k_{\mathrm{x}}^{2}=\frac{\left(\omega_{\mathrm{d}}^{2}-k_{\mathrm{z}}^{2} c_{\mathrm{A}}^{2}\right)\left(\omega_{\mathrm{d}}^{2}-k_{\mathrm{z}}^{2} v_{\mathrm{S}}^{2}\right)}{\omega_{\mathrm{d}}^{2}\left(c_{\mathrm{A}}^{2}+v_{\mathrm{S}}^{2}\right)-k_{\mathrm{z}}^{2} v_{\mathrm{S}}^{2} c_{\mathrm{A}}^{2}}$

This well-known equation is used in different papers while discussing the K.-H. instability (e.g. Southwood, 1968; Pu and Kivelson, 1983). It should be mentioned that Eq. (11) contains several particular points where

$\omega_{\mathrm{d}}^{2}(x)=k_{\mathrm{z}}^{2} c_{\mathrm{A}}^{2}, \quad \omega_{\mathrm{d}}^{2}(x)=k_{\mathrm{z}}^{2} v_{\mathrm{S}}^{2}, \quad \omega_{\mathrm{d}}^{2}(x)=\frac{k_{\mathrm{z}}^{2} c_{\mathrm{A}}^{2} v_{\mathrm{S}}^{2}}{c_{\mathrm{A}}^{2}+v_{\mathrm{S}}^{2}}$

In the vicinity of these points the approximation of the geometric optics is not valid. So, a more accurate investigation is required.

We suppose that the speed $v_{\mathrm{p}}(x)$ changes monotonously with $x$ from $v_{\mathrm{p}}=0$ in the magnetosphere $(x<-d)$ to $v_{\mathrm{p}}=V_{0}$ in the magnetosheath $(x>d)$. It follows from relations (13) that in general there are six particular points $x_{1}^{ \pm}, x_{2}^{ \pm}, x_{3}^{ \pm}$for which

$$
\begin{aligned}
& k_{\mathrm{z}} v_{\mathrm{p}}\left(x_{1}^{ \pm}\right)=\omega \pm \frac{k_{\mathrm{z}} v_{\mathrm{S}}\left(x_{1}^{ \pm}\right) c_{\mathrm{A}}\left(x_{1}^{ \pm}\right)}{\sqrt{v_{\mathrm{S}}^{2}\left(x_{1}^{ \pm}\right)+c_{\mathrm{A}}^{2}\left(x_{1}^{ \pm}\right)}}, \\
& k_{\mathrm{z}} v_{\mathrm{p}}\left(x_{2}^{ \pm}\right)=\omega \pm k_{\mathrm{z}} c_{\mathrm{A}}\left(x_{2}^{ \pm}\right), k_{\mathrm{z}} v_{\mathrm{p}}\left(x_{3}^{ \pm}\right)=\omega \pm k_{\mathrm{z}} v_{\mathrm{S}}\left(x_{3}^{ \pm}\right)
\end{aligned}
$$

At the first two particular points $x_{1}^{ \pm}$the SM wave propagates perpendicular to the direction of flow. Indeed, according to Eq. (12) at $x=x_{1}^{ \pm}$the absolute value of the wave number $k_{\mathrm{x}}$ tends to infinity, that is $k_{\mathrm{x}}^{2} \gg k_{\mathrm{z}}^{2}$. The relation

$\omega_{\mathrm{d}}^{2}=\frac{k_{\mathrm{z}}^{2} v_{\mathrm{S}}^{2} c_{\mathrm{A}}^{2}}{v_{\mathrm{S}}^{2}+c_{\mathrm{A}}^{2}}$

coincides with the dispersion relation for the SM wave in the transverse case $k_{\mathrm{x}}^{2} \gg k_{\mathrm{z}}^{2}$. The other particular points $x_{2}^{ \pm}$and $x_{3}^{ \pm}$correspond to the SM and FM waves in the case when $k_{\mathrm{x}} \approx 0$. It is convenient to introduce a parameter $\beta=v_{\mathrm{S}}^{2} / c_{\mathrm{A}}^{2}$, which is equal to the ratio of the thermal pressure to the magnetic pressure. For a positive sign on the right-hand sides of Eqs. (14) the particular points are distributed along the $\mathrm{x}$-axis in the following manner $x_{1}^{+}<x_{2}^{+}<x_{3}^{+}$. At the flank-side magnetopause, where $x_{1}^{+}<x<x_{2}^{+}$, the plasma is transparent for propagation. According to the analysis presented above, the SM wave can be present in this region. Its amplitude decreases outwards for $x>x_{2}^{(+)}$and $x<x_{1}^{(+)}$. Hence, this SM wave can be approximately considered as a surface wave. For negative signs another relation holds depending on the magnitude of the parameter $\beta$. If $\beta$ at the inner side of the magnetopause $\beta_{\mathrm{sp}}$ is also larger than unity, the particular points are distributed in the following manner: $x_{3}^{-}<x_{2}^{-}<x_{1}^{-}$. In this case, the SM wave can be excited in the region $x_{2}^{-}<x<x_{1}^{-}$. Note, that in the general case there is a link between the SM waves existing in two different regions: $x_{1}^{+}<x<x_{2}^{+}$and $x_{2}^{-}<x<x_{1}^{-}$. This link can be very small (the waves are practically independent) if the wave attenuation in the region $x_{1}^{-}<x<x_{1}^{+}$is large enough. The attenuation depends on the parameters of the medium and the excited wavelength. If $\beta_{\mathrm{sp}}$ is less than unity (which is more typical), we have $x_{2}^{-}<x_{3}^{-}<x_{1}^{-}$. The SM wave in this case is localized in the region $x_{3}^{-}<x<x_{1}^{-}$. Note that the wave propagation, in the form of a FM wave, is possible in the region $x>x_{3}^{+}$for the supersonic velocity $V_{0}$ when the absolute value of the frequency $\omega_{\mathrm{d}}$ exceeds $k_{\mathrm{z}} v_{\mathrm{S}}$. In the present paper, we shall discuss separately two cases. First, we consider the case when the FM waves are not excited in the magnetosheath $\left(\omega_{\mathrm{d}}^{2}<k_{\mathrm{z}}^{2} v_{\mathrm{S}}^{2}\right)$ and the surface waves are present at the magnetopause (the usual K.H. instability). Secondly, we analyse the situation when the FM wave is excited at the magnetopause. Due to this the K.$\mathrm{H}$. instability becomes more complicated and involves, under some conditions (see Sect. 6), the propagation of FM waves into the magnetosheath.

\section{Kelvin-Helmholtz instability at the nightside magnetopause}

It is convenient to introduce the dimensionless frequency $f_{\mathrm{d}}=\frac{\omega_{\mathrm{d}}}{k_{\mathrm{z}} c_{\mathrm{A}}}$, the velocity $u=\frac{v_{\mathrm{p}}}{k_{\mathrm{z}} c_{\mathrm{A}}}$ and the dimensionless coordinate $\rho=k_{\mathrm{Z}} x$. Let us assume that parameter $\beta$ is a constant quantity and large enough $(\beta>1)$ and at the same time the flow velocity is not too high. Then in the vicinity of the magnetopause (if we neglect the excitation of the KA wave) a common surface wave is formed. This means that the amplitude of the magnetosonic perturbation tends towards zero with increasing distance from the magnetopause. In other words, no FM wave is excited in the magnetosheath and in the magnetosphere due to perturbations at the magnetopause. In Eq. (11), there are no singularities because $\omega_{\mathrm{d}}^{2}<k_{\mathrm{z}}^{2} v_{\mathrm{S}}^{2}$ everywhere. Due to this, we may simplify Eq. (11) by assuming that $\omega_{\mathrm{d}}^{2} \ll k_{\mathrm{z}}^{2} v_{\mathrm{S}}^{2}$

$$
\frac{\partial}{\partial \rho}\left(f_{\mathrm{d}}^{2}-\frac{\beta}{\beta+1}\right) \frac{\partial A_{\mathrm{y}}}{\partial \rho}-\frac{\beta}{\beta+1}\left(f_{\mathrm{d}}^{2}-1\right) A_{\mathrm{y}}=0
$$


Now we multiply Eq. (15) by $A_{\mathrm{y}}^{*}$ and integrate it with respect to $\rho$. Taking into account that $A_{\mathrm{y}} \rightarrow 0$ if $|\rho| \rightarrow \infty$ we find

$$
\begin{aligned}
& \int_{-\infty}^{\infty}\left(f_{\mathrm{d}}^{2}-\frac{\beta}{\beta+1}\right)\left|\frac{\partial A_{\mathrm{y}}}{\partial \rho}\right|^{2} d \rho \\
& \quad+\frac{\beta}{\beta+1} \int_{-\infty}^{\infty}\left(f_{\mathrm{d}}^{2}-1\right)\left|A_{\mathrm{y}}\right|^{2} d \rho=0
\end{aligned}
$$

Let us single out the averaged velocity $<u>$

$$
<u>=\frac{\left.\int u\left(\left|\frac{\partial A_{\mathrm{y}}}{\partial \rho}\right|^{2}+\frac{\beta}{\beta+1}\left|A_{\mathrm{y}}\right|^{2}\right)\right) d \rho}{\left.\int\left(\left|\frac{\partial A_{\mathrm{y}}}{\partial \rho}\right|^{2}+\frac{\beta}{\beta+1}\left|A_{\mathrm{y}}\right|^{2}\right)\right) d \rho}
$$

If we introduce the dimensionless frequency $f_{1}=f-<u>$ and the velocity $u_{1}=u-<u>$, Eq. (16) near the threshold of instability can be expressed in the form

$f_{1}^{2}+<u_{1}^{2}>-\frac{B}{A}=0$,

where

$$
\begin{gathered}
\left.A=\int\left(\left|\frac{\partial A_{\mathrm{y}}}{\partial \rho}\right|^{2}+\frac{\beta}{\beta+1}\left|A_{\mathrm{y}}\right|^{2}\right)\right) d \rho \\
B=\frac{\beta}{\beta+1} \int\left(\left|\frac{\partial A_{\mathrm{y}}}{\partial \rho}\right|^{2}+\left|A_{\mathrm{y}}\right|^{2}\right) d \rho
\end{gathered}
$$

It is clear from Eq. (18) that the instability appears if $\left.<u_{1}^{2}\right\rangle$ exceeds $B / A$. It means that the square of the characteristic velocity $u_{1}$ should be of the order of unity or more. This condition determines the threshold of instability $v_{c, 1}$. Note that at the threshold of instability the dimensionless frequency of perturbations is $f=<u>$. This means that the frequency in the stationary (magnetospheric) reference frame is $\omega=k_{\mathrm{z}} c_{\mathrm{A}}<u>$.

Let us now make some estimates. Suppose that the velocity $v_{\mathrm{p}}(x)$ changes with $x$ according to

$v_{\mathrm{p}}(x)=0.5 V_{0}\left[1+\tanh \left(\frac{x}{d}\right)\right]$

From this equation, we find the typical gradient of the flow velocity along the $\mathrm{x}$-axis $d v_{\mathrm{p}} / d x \approx 0.5 V_{0} / d$. If only this flow velocity changes at the magnetopause and the other parameters (temperature, plasma density, magnetic field) are constant quantities, then due to the symmetry $\langle u\rangle=0.5 V_{0}$. The integrals (19) in Eq. (18) can be calculated if we choose a specific distribution of the potential $A_{\mathrm{y}}$ and its derivative as functions of the $\mathrm{x}$-coordinate. For this purpose, we need to match the solutions in the vicinities of the particular points with the solution in the geometric optics approximation valid far away from these points.

To estimate $<u_{1}^{2}>$ we apply the geometric optics approximation and calculate the input of the region $x \geq x_{2}^{+}$. For the case $2 c_{\mathrm{A}}<V_{0}<2 v_{S}$, assuming that the region $x \geq x_{2}^{+}$ provides the main contribution, we find

$$
\begin{aligned}
& <u_{1}^{2}>\approx \frac{V_{0}^{2}}{4 c_{\mathrm{A}}^{2}}, \quad k_{\mathrm{Z}} d<1 \\
& <u_{1}^{2}>\approx \frac{V_{0}^{2}}{4 c_{\mathrm{A}}^{2}} \frac{1.25}{k_{\mathrm{Z}}^{2} d^{2}}, \quad k_{\mathrm{Z}} d>1
\end{aligned}
$$

Now let us discuss another case when the flow velocity is supersonic $\left(V_{0}>2 v_{\mathrm{S}}\right)$. This situation is typical for the flanks of the Earth's magnetosheath and the magnetosheath of Mars or Venus. In this case, our main Eq. (11) contains a singularity at the point $x_{3}^{+}$where $\omega_{\mathrm{d}}(x)=-k_{\mathrm{z}} v_{\mathrm{S}}$. This means that the FM wave is excited at this point and we do not have a pure surface wave. Note that previously the excitation of the kinetic Alfvén wave at the singular point was discussed by Hasegawa and Chen (1976). Ohsawa et al. (1976) mentioned that such an excitation influences the growth rate of the K.-H. instability. But a detailed analysis was not presented.

We shall use a somewhat different approach than was utilized in the papers cited above and start with Eq. (11). Let us integrate this equation within a finite range of coordinates $\rho_{\min }<\rho<\rho_{\max }$. For the upper boundary, we take the point $\rho_{\max }=k_{\mathrm{z}} x_{3}^{+}$and for the lower boundary $\rho_{\min }=k_{\mathrm{z}} x_{3}^{-}$ if $\beta_{\mathrm{sp}}>1$ and $\rho_{\min }=k_{\mathrm{z}} x_{2}^{-}$if $\beta_{\mathrm{sp}}<1$. Assuming once again that $L_{v} / L_{N} \approx 0$, we arrive at the equation that determines the instability

$$
\begin{aligned}
\int_{\rho_{\min }}^{\rho_{\max }} & \frac{(\beta+1) f_{\mathrm{d}}^{2}-\beta}{f_{\mathrm{d}}^{2}-\beta}\left|\frac{\partial A_{\mathrm{y}}}{\partial \rho}\right|^{2} d \rho \\
& -\int_{\rho_{\min }}^{\rho_{\max }}\left(f_{\mathrm{d}}^{2}-1\right)\left|A_{\mathrm{y}}\right|^{2} d \rho- \\
& -\left.\frac{(\beta+1) f_{\mathrm{d}}^{2}-\beta}{f_{\mathrm{d}}^{2}-\beta} A_{\mathrm{y}}^{*} \frac{\partial A_{\mathrm{y}}}{\partial \rho}\right|_{\rho_{\max }} \\
& +\left.\frac{(\beta+1) f_{\mathrm{d}}^{2}-\beta}{f_{\mathrm{d}}^{2}-\beta} A_{\mathrm{y}}^{*} \frac{\partial A_{\mathrm{y}}}{\partial \rho}\right|_{\rho_{\min }}=0
\end{aligned}
$$

The third term in Eq. (22) describes the influence of the outgoing FM wave on the instability. Similarly the fourth term describes the influence of the magnetosonic wave (in the case if it is exited) propagating from the magnetopause into the magnetosphere. It is seen that the denominator in the first, the third and the fourth term in Eq. (22) tends to zero if $\rho_{\max } \rightarrow k_{\mathrm{z}} x_{3}^{+}$and $\rho_{\min } \rightarrow k_{\mathrm{z}} x_{3}^{-}$. But at the same time the numerator also tends to zero. For the point $x_{3}^{+}$this can be verified if we substitute the solution (A14) obtained in the Appendix into Eq. (22). For the lower boundary this can be shown similarly.

In this paper, we shall discuss only the matching of solutions at the upper boundary because we are interested in the problem how the FM wave propagating into the magnetosheath influences the instability. If only the flow velocity is changed across the magnetopause the input to Eq. (22) from the lower (magnetospheric) boundary due to the symmetry is similar as from the upper boundary. If other parameters are also changed across the magnetopause, the input of the 
magnetospheric boundary should be taken into account in an explicit form. It means that in a general case the K.-H. instability at the magnetopause is influenced simultaneously by both boundaries (the upper and the lower).

Let us assume now that $V_{0}>2 v_{\mathrm{S}}$. Our aim is to investigate how the outgoing FM wave influences the growth rate of the K.-H. instability. It is convenient to introduce two Mach numbers, the Alfvénic one $M_{\mathrm{A}}=\frac{V_{0}}{c_{\mathrm{A}}}$ and the sonic Mach number $M_{\mathrm{S}}=\frac{V_{0}}{v_{\mathrm{s}}}$. As the fluid velocity $V_{0}$ is supposed to be supersonic, we shall assume that $M_{\mathrm{A}} \gg 1$ and $M_{\mathrm{S}}>2$.

In this section, we neglect the excitation of the kinetic Alfvén wave (which is treated in Sect. 5). Also to simplify our calculations, we assume that all parameters except the flow velocity do not change across the magnetopause. Taking into account Eq. (22) it is convenient to introduce the following quantities

$$
\begin{aligned}
& I_{1} \approx \frac{V_{0}^{2}}{c_{\mathrm{A}}^{2}} \int_{k_{\mathrm{z}} L_{v}}^{\sqrt{\beta_{s}} k_{\mathrm{z}} L_{v}}\left(-\frac{\beta+1}{f_{\mathrm{d}}^{2}-\beta}\left|\frac{\partial A_{\mathrm{y}}}{\partial \rho}\right|^{2}+\left|A_{\mathrm{y}}\right|^{2}\right) \tanh ^{2}\left(\frac{\rho}{k_{\mathrm{z}} d}\right) d \rho, \\
& I_{2} \approx \int_{k_{\mathrm{z}} L_{v}}^{\sqrt{\beta} k_{\mathrm{z}} L_{v}}\left(-\frac{\beta}{f_{\mathrm{d}}^{2}-\beta}\left|\frac{\partial A_{\mathrm{y}}}{\partial \rho}\right|^{2}+\left|A_{\mathrm{y}}\right|^{2}\right) d \rho,
\end{aligned}
$$$$
I_{3}=-\left.\frac{(\beta+1) f_{\mathrm{d}}^{2}-\beta}{f_{\mathrm{d}}^{2}-\beta_{s}} A_{\mathrm{y}}^{*} \frac{\partial A_{\mathrm{y}}}{\partial \rho}\right|_{\rho_{\max }}
$$

$I_{4}=\left.\frac{(\beta+1) f_{\mathrm{d}}^{2}-\beta}{f_{\mathrm{d}}^{2}-\beta_{s}} A_{\mathrm{y}}^{*} \frac{\partial A_{\mathrm{y}}}{\partial \rho}\right|_{\rho_{\text {min }}}$

Neglecting the excitation of the FM wave, we find, with the help of Eq. (23), the condition for the K.-H. instability takes the form

$$
I_{1}-I_{2}-\frac{2}{3} k_{\mathrm{z}} L_{v}\left|C_{0}\right|^{2}>0
$$

This condition means that the instability appears if the flow velocity exceeds the critical value. (The first term $I_{1}$ exceeds the contribution of the two other terms). The third term $\frac{2}{3} k_{\mathrm{z}} L_{v}\left|C_{0}\right|^{2}$ in Eq. (24) corresponds to the input of the region $0<x<x_{1}^{+}$.

When the FM wave is excited, the term $I_{3}$ gives a finite contribution to the growth rate of the instability. It can be verified that in the case where only the flow velocity changes across the magnetopause the contribution of the real parts of the terms $I_{3}$ and $I_{4}$ compensate each other. The imaginary parts give the same contribution $\operatorname{Im}\left(I_{3}\right)$ to Eq. (22). But in the general case the contribution of the terms $I_{3}$ and $I_{4}$ to Eq. (22) is different. For example, if $k_{\mathrm{z}}\left(x_{1}^{+}-x_{1}^{-}\right) \gg 1$ there is practically no connection between the lower (magnetospheric) and the upper parts of the magnetopause. In such a case, the term $I_{4}$ almost does not influence the K.-H. instability excited at the upper part of the magnetopause. It means that in general two different regions $x_{1}^{+}<x<x_{2}^{+}$and $x_{2}^{-}<x<x_{1}^{-}$can give rise to two K.-H. instabilities (at the outer boundary of the magnetopause and at the inner boundary). For small-scale perturbations $k_{\mathrm{z}}\left(x_{1}^{+}-x_{1}^{-}\right) \gg 1$ when the connection between two regions is very small only the instability at the inner boundary is connected with processes in the magnetosphere. At the same time, the instability at the outer boundary can be the source of the LF waves in the magnetosheath.

Let us introduce

$\sin \phi=\frac{\rho}{\sqrt{\beta} k_{\mathrm{z}} L_{v}}$, where $L_{v}=\frac{2 d c_{\mathrm{A}}}{V_{0}}$

In the case $k_{\mathrm{z}} d>1$ after substitution in Eqs. (23) of the electric potential $A_{\mathrm{y}}$ found in the Appendix, we arrive at the approximate contribution of the main terms

$$
\begin{aligned}
I_{1}= & 2\left|C_{3}\right|^{2} \exp (P) \beta^{3 / 2} L_{\mathrm{S}} \int_{1 / \sqrt{\beta}}^{\pi / 2}(\cos \phi)^{2}(\sin \phi)^{2} \\
& \exp \left[-L_{\mathrm{S}}\left(\phi+\sin \phi \cos \phi-\frac{2}{\sqrt{\beta}}\right)\right] d \phi, \\
I_{2}= & \left|C_{3}\right|^{2} \exp (P) \beta^{1 / 2}\left(\frac{\beta}{\beta+1}+1\right) L_{\mathrm{S}} \int_{1 / \sqrt{\beta}}^{\pi / 2}(\cos \phi)^{2} \\
& \exp \left[-L_{\mathrm{S}}\left(\phi+\sin \phi \cos \phi-\frac{2}{\sqrt{\beta}}\right)\right] d \phi, \\
I_{3}= & 0.92\left|C_{3}\right|^{2} \beta\left(\left(\cos \frac{2 \pi}{3}\right)-i \sin \left(\frac{2 \pi}{3}\right)\right)
\end{aligned}
$$

Here $L$ is a dimensionless parameter $L=2 d k_{\mathrm{z}} / M_{\mathrm{S}}$. It follows from Eqs. (25) that in the limiting case $k_{\mathrm{z}} d>1$ there are only two main parameters $\beta$ and $L$ that determine the threshold of instability (if the flow velocity is parallel to the magnetic field). For finite values of $L, \beta$ and $k_{\mathrm{z}} d$ the contribution of different terms can be evaluated numerically. Note, that for estimates we have only used the one exponent $\exp P$ in Eq. (25). This is correct if the attenuation is high enough $P \geq 1$. For small values of $L$, two exponents (increasing and decreasing) should be retained in Eq. (25). That is why our results for $L<1$ can be considered only as a rather crude approximation.

Assume that the threshold of the K.-H. instability is exceeded even without taking into account the FM wave. Our aim is to find out how the growth rate is influenced by the excitation of the FM wave leaking into the magnetosheath. We suppose that only the flow velocity changes across the magnetopause. In this case the real parts of $I_{3}$ and $I_{4}$ at the upper and the lower boundaries of integration compensate each other and the imaginary part $\operatorname{Im}\left(I_{3}\right)=\operatorname{Im}\left(I_{4}\right)$ gives the contribution to the growth rate. The corresponding growth rate is given by the real part of $G$, where

$G \approx \frac{1}{M_{\mathrm{s}}}\left(\frac{3\left(I_{1}-I_{2}-i \operatorname{Im}\left(I_{3}\right)\right)-2 L}{3\left(I_{2}+L\right)}\right)^{1 / 2}$,

In Fig. 1, the dimensionless growth rates $\operatorname{Re} G(L)$ (normalized to $\left.c_{\mathrm{A}} / 2 d\right)$ for $M_{\mathrm{S}}=3, \beta=3.5$ are presented. The broken line shows the growth rate calculated without taking into account the FM wave excitation. The continuous line shows the growth rate calculated from Eq. (26). One can see that 


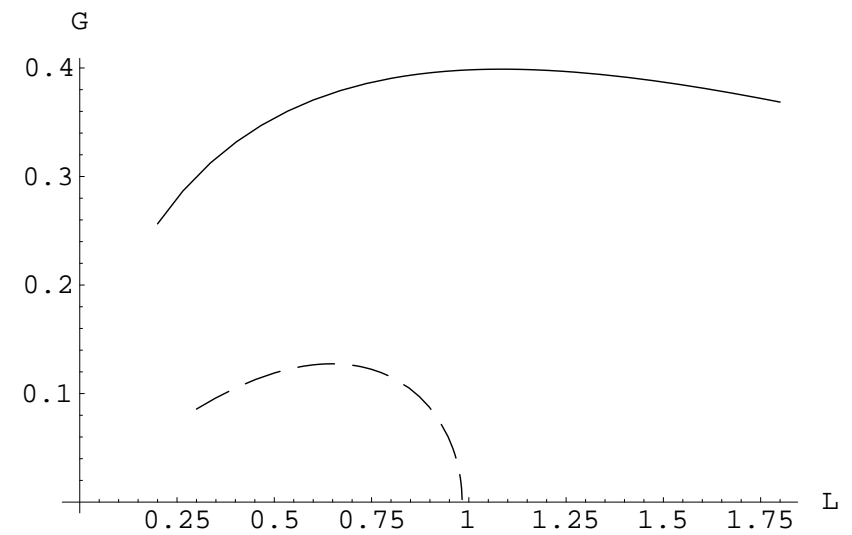

Fig. 1. The growth rate $G$ of the K.-H. instability (normalized to $c_{\mathrm{A}} / 2 d$ ) for the the sonic Mach number $M_{\mathrm{S}}=3$, plasma pressure $\beta=3.5$ is presented as a function of the dimensionless parameter $L=2 d k_{\mathrm{z}} / M_{\mathrm{S}}$. The growth rate of instability taking into account the excitation of the FM wave propagating into the magnetosheath is given by a continuous line. The growth rate of instability neglecting the excitation of the FM wave is presented by a broken line.

the excitation of the FM wave propagating into the magnetosheath raises very strongly the growth rate of the K.-H. instability due to the negative energy of the FM wave (see Sect. 6).

We can estimate the perturbed longitudinal magnetic field $b_{\|}$of the excited FM wave in the magnetosheath $x>x_{3}^{+}$with respect to the field of the SM wave at the point $x=x_{2}^{+}$with the help of the results obtained above:

$b_{\|}(x) \approx \exp (-0.5 P) b_{\|}\left(x_{2}^{+}\right)$

Note, that due to the excitation of the K.-H. instability all perturbations in the linear approximation grow exponentially in time.

\section{Excitation of the kinetic Alfvén wave}

The idea that kinetic Alfvén (KA) waves are generated due to the linear transformation of the magnetosonic waves which in turn appear as a result of the excitation of the K.-H. instability was introduced many years ago (Ohsawa et al., 1976). Ohsawa et al. (1976) have derived an equation describing the KA wave in a low-beta $(\beta \ll 1)$ plasma. For high-beta plasma $(\beta>1)$ the corresponding equation was obtained by Mikhailovskii and Onishchenko (1995). Unfortunately, in Ohsawa et al. (1976) and Mikhailovskii and Onishchenko (1995) the transverse electric field (with respect to the background magnetic field $\boldsymbol{B}_{0}$ ) was expressed as $\boldsymbol{E}_{\perp}=-\nabla \phi$, where $\phi$ is the electric potential. In this approximation, the interaction between KA and magnetosonic waves can not be taken into account appropriately. Indeed only the transverse electric field of the Alfvén wave (and not of the magnetosonic wave) is of a potential type $\boldsymbol{E}_{\perp}=-\nabla \phi$. To discuss the interaction of magnetosonic and Alfvén waves in the general case, three components of the wave vector $\left(k_{\mathrm{x}}, k_{\mathrm{y}}, k_{\mathrm{z}}\right)$ should be taken into account. As a result a differential equation that describes two types of resonances (Alfvénic and magnetosonic) is obtained (see, e.g. Taroyan and Erdélyi, 2002). In our case $\left(k_{\mathrm{y}}=0\right)$ the Alfvén resonance disappears. But still the excitation of Alfvén waves is possible. This problem is discussed as follows.

In the 2-D case the Alfvén wave has a transverse $E_{\mathrm{x}}$ component while the magnetosonic waves have only an $E_{\mathrm{y}}$ transverse electric field component. According to Ohsawa et al. (1976) and Mikhailovskii and Onishchenko (1995) the dispersion relation for KA waves in the geometric optics approximation takes the form

$\omega^{2}=k_{\mathrm{z}}^{2} c_{\mathrm{A}}^{2}\left[1+k_{\mathrm{x}}^{2} \rho_{\mathrm{eff}}^{2}\right]$,

where

$\rho_{\mathrm{eff}}^{2}=0.75 \rho_{\mathrm{Hi}}^{2}+\rho_{*}^{2}, \quad \rho_{\mathrm{Hi}}=\frac{T_{\mathrm{i}}}{M \omega_{\mathrm{Hi}}}, \quad \rho_{*}=\frac{r_{\mathrm{D}} c}{c_{\mathrm{A}}}$

$\rho_{\mathrm{Hi}}$ is the Larmor radius of ions, $r_{\mathrm{D}}$ is the Debye radius. This equation can be obtained either in kinetics (see Ohsawa et al., 1976) or in two-fluid magnetohydrodynamics taking into account the tensor of collisionless viscosity $\{\pi\}$ (see Mikhailovskii and Onishchenko, 1995).

The aim of this section is to obtain a differential equation describing the excitation of the KA wave in the inhomogeneous plasma in which the interaction with the magnetosonic waves is taken into account.

In the case of a moving plasma, the dispersion relation for the KA wave is easily obtained from Eq. (28)

$\omega_{\mathrm{d}}^{2}=k_{\mathrm{z}}^{2} c_{\mathrm{A}}^{2}\left[1+k_{\mathrm{x}}^{2} \rho_{\mathrm{eff}}^{2}\right]$

It is clear from Eq. (29) that the KA wave exists for the frequencies $\left|\omega_{\mathrm{d}}\right| \geq k_{\mathrm{Z}} c_{\mathrm{A}}$. In contrast to the ordinary Alfvén wave the kinetic one has a component of the group velocity across the magnetic field (due to the term $k_{\mathrm{x}}^{2} \rho_{\mathrm{eff}}^{2}$ ). It means that the KA wave, if generated at the magnetopause, can propagate into the magnetosheath even if the background magnetic field is parallel to the flow velocity.

We start with the $\mathrm{x}$-component of the Maxwell equation for the electric field. On the right-hand side of this equation the electric current generating this field should be expressed in an explicit form:

$$
\frac{\partial}{\partial x}(\nabla \cdot \boldsymbol{E})-\Delta E_{\mathrm{x}}=-\frac{4 \pi i \omega_{\mathrm{d}}}{c^{2}} e N_{0}\left(v_{i, x}-v_{e, x}\right)
$$

As the electric current $e N_{0}\left(v_{i, x}-v_{e, x}\right)$ in the magnetized plasma depends not only on $E_{\mathrm{x}}$, but also on the $E_{\mathrm{y}}$ component of the electric field, the link between the two types of waves (Alfvén and magnetosonic) appears. We suppose that in the region where the interaction of the Alfvén and the magnetosonic waves takes place the variations of the density $N_{0}$, 
the temperatures $T_{\mathrm{i}}, T_{\mathrm{e}}$ and the magnetic field $\boldsymbol{B}_{0}$ along the $\mathrm{x}$-axis are much weaker than the dependence of the fluid velocity $v_{\mathrm{p}}(x)$ on the $\mathrm{x}$-coordinate. In this approximation, after some transformations in the vicinity of the Alfvén resonance region $\omega_{\mathrm{d}}^{2} \approx k_{\mathrm{Z}}^{2} c_{\mathrm{A}}^{2}$, we arrive at the equation

$k_{\mathrm{z}}^{2} \rho_{\mathrm{eff}}^{2} \frac{\partial^{2} E_{\mathrm{x}}}{\partial x^{2}}+\left(\frac{\omega_{\mathrm{d}}^{2}}{c_{\mathrm{A}}^{2}}-k_{\mathrm{z}}^{2}\right) E_{\mathrm{x}}=Q(x, t)$,

where $Q(x, t)$ is the source for the KA wave

$$
Q(x, t) \approx-i \frac{\omega_{\mathrm{d}}^{3}}{c_{\mathrm{A}}^{2} \omega_{\mathrm{Hi}}} E_{\mathrm{y}}
$$

In this source, we have neglected terms on the order of $k_{\mathrm{x}}^{2} \rho_{\mathrm{Hi}}^{2}$ which are assumed to be small enough. Note, that the source $Q(x, t)$ grows with time due to the K.-H. instability. In the linear approximation, the growth is described by exp $\gamma t$, where $\gamma$ is the growth rate of the K.-H. instability. It follows from Eqs. (31), (32) that the coupling between KA and magnetosonic waves has the order of magnitude $\sim \frac{\omega_{\mathrm{d}}}{\omega_{\mathrm{Hi}}}$. In the present paper, this parameter is considered as small: $\frac{\omega_{\mathrm{d}}}{\omega_{\mathrm{Hi}}} \ll 1$. Nevertheless assuming that the surface waves are intensive enough due to the K.-H. instability, the excited KA wave can also acquire significant amplitude.

In the vicinity of the interaction region, the frequency $\omega$ can be expressed as

$\omega=k_{\mathrm{z}} v_{\mathrm{p}}(x)-k_{\mathrm{z}} c_{\mathrm{A}}\left(1+\frac{1}{2} k_{\mathrm{x}}^{2} \rho_{\mathrm{eff}}^{2}\right)$

This frequency is a constant quantity. As the flow velocity increases towards the magnetosheath, the corresponding $k_{\mathrm{x}}$ number should also increase in this direction. Note that the excited KA wave has a negative frequency in the reference frame moving with the plasma. Its group velocity along the $\mathrm{x}$-axis is

$V_{g, x} \approx-k_{\mathrm{z}} c_{\mathrm{A}} k_{\mathrm{x}} \rho_{e f f}^{2}$

This means that the $\mathrm{x}$-component of the group velocity is directed into the magnetosheath only for negative values of $k_{\mathrm{x}}$.

It is convenient to introduce a new dimensionless variable

$\tau(x)=\frac{v_{0}^{1 / 3}\left(x-x_{2}^{+}\right)}{\left(c_{\mathrm{A}} \rho_{\mathrm{eff}}^{2} d\right)^{1 / 3}}$

As a result, Eq. (31) can be expressed in the form of an equation for the Airy function

$\frac{\partial^{2} E_{\mathrm{X}}}{\partial \tau^{2}}+\tau E_{\mathrm{x}}=\frac{\left(c_{\mathrm{A}} d\right)^{2 / 3}}{k_{\mathrm{Z}}^{2}\left(V_{0} \rho_{\mathrm{eff}}\right)^{2 / 3}} Q$

Equation (36) has an oscillating solution for $\tau>0$ and exponential (decreasing or increasing) solutions in the region $\tau<0$. We are interested in a solution that describes the wave propagating into the magnetosheath for $x>x_{2}^{+}$and the exponentially decreasing wave in the region $x<x_{2}^{+}$. To find the amplitude of the excited Alfvén wave, we need to introduce the Green function for Eq. (36) that corresponds to the outgoing wave for $\tau>0$ and the attenuated wave for $\tau<0$

$$
\begin{aligned}
G(\tau>0)= & \frac{1}{\Delta} \tau^{1 / 2} H_{1 / 3}^{(1)}\left(\frac{2}{3} \tau^{3 / 2}\right) \\
G(\tau<0)= & \frac{1}{\Delta}\left(-\tau^{1 / 2}\right)\left[-I_{1 / 3}\left(-\frac{2}{3} \tau^{3 / 2}\right)\right. \\
& \left.+I_{-1 / 3}\left(-\frac{2}{3} \tau^{3 / 2}\right)\right]
\end{aligned}
$$

Here $H_{1 / 3}^{(1)}$ is the Hankel function of the order $1 / 3, I_{1 / 3}$ is a modified Bessel function of the same order, $\Delta$ is a Wronskian. With the help of the Green function (37), we express the solution of the inhomogeneous Eq. (31) in the form

$E_{\mathrm{X}}(\tau)=\frac{d^{2 / 3}}{M_{\mathrm{A}}^{2 / 3} k_{\mathrm{Z}}^{2} \rho_{\mathrm{eff}}^{2 / 3}} \int Q\left(\tau_{1}\right) G\left(\tau-\tau_{1}\right) d \tau_{1}$

This equation allows us to estimate the amplitude of the KA wave due to the transformation of the slow magnetosonic oscillations in the inhomogeneous plasma. Such an estimate is easily obtained with the help of Eqs. (37)-(38)

$\frac{E_{\mathrm{x}}}{E_{\mathrm{y}}\left(x_{2}^{+}\right)} \approx \frac{k_{\mathrm{z}} c_{\mathrm{A}} d^{2 / 3}}{M_{\mathrm{A}}^{2 / 3} \rho_{\mathrm{Hi}}^{2 / 3} \omega_{\mathrm{Hi}}}$

Here $E_{\mathrm{y}}\left(x_{2}^{+}\right)$is the amplitude of the SM wave at the point $x=x_{2}^{+}$.

\section{Propagation of the FM waves in a weakly inhomogeneous magnetosheath}

While discussing the K.-H. instability, it is often assumed that the background plasma is inhomogeneous only in one direction (along the $\mathrm{x}$-axis) and the medium along two other axes is homogeneous. In reality this is not the case. Parameters of the medium (magnetic field, plasma density and temperature are changing not only across the magnetosheath, but also rather weakly along it (towards the tail). To take into account such changes is important because it allows us to find out in what parts of the magnetopause the K.-H. instability can be excited (see, e.g. Takagi et al., 2006) and where the generated waves can be detected. The aim of this section is to derive equations in the geometric optics approximation which describe the propagation of the FM waves in a weakly inhomogeneous magnetosheath. We shall investigate the propagation of the wave packet outside the region of excitation. The collisionless attenuation is assumed to be small enough. 
According to Eq. (12) the dispersion relation for the FM wave near the region of its generation $\omega_{\mathrm{d}} \approx-k_{\mathrm{z}} v_{\mathrm{S}}$ in a plasma with $\beta>1$ can be expressed in the form

$\omega=k_{\mathrm{z}}\left(v_{\mathrm{p}}-v_{\mathrm{S}}\right)-\frac{k_{\mathrm{x}}^{2}}{2 k_{\mathrm{z}}} \frac{v_{\mathrm{S}}^{3}}{v_{\mathrm{S}}^{2}-c_{\mathrm{A}}^{2}}$

With the help of Eq. (40), it becomes clear that in the geometric optics approximation all perturbations change in space and in time according to

$$
\begin{gathered}
\propto \exp \left[i k_{\mathrm{z}}\left(v_{\mathrm{p}}-v_{S}\right) t-i \frac{k_{\mathrm{x}}^{2}}{2 k_{\mathrm{z}}} \frac{v_{S}^{3}}{v_{S}^{2}-c_{\mathrm{A}}^{2}} t\right. \\
\left.-i \int k_{\mathrm{x}}\left(x_{1}\right) d x_{1}-i \int^{z} k_{\mathrm{z}} d z_{1}\right]
\end{gathered}
$$

It follows from Eq. (41) that in the system of coordinates moving with the plasma, the excited FM wave has negative frequency while in the motionless reference frame it has positive frequency. This frequency given by Eq. (40) should be constant in the magnetospheric system of coordinates. It means that the propagation of the FM wave packet into the magnetosheath is possible if the difference of the velocities $v_{\mathrm{p}}-v_{\mathrm{S}}$ is positive along the trajectory of the wave packet. Note that in the case if the main plasma parameters change in both directions (along the $\mathrm{x}$-axis and the $\mathrm{z}$-axis), the $\mathrm{k}$ numbers $\left(k_{\mathrm{x}}, k_{\mathrm{z}}\right)$ also change in space. To find the wave numbers in the inhomogeneous plasma, we introduce the phase $S$ along the trajectory in such a way that

$k_{\mathrm{x}}=\frac{\partial S}{\partial x}, \quad k_{\mathrm{z}}=\frac{\partial S}{\partial z}$

The phase $S$ is determined by following equation (compare with Eq. 40):

$v_{\mathrm{S}}^{2}(\mathbf{r})\left(\frac{\partial S}{\partial z}\right)^{2}+\frac{v_{\mathrm{S}}^{4}(\mathbf{r})}{\left(v_{\mathrm{S}}^{2}(\mathbf{r})-c_{\mathrm{A}}^{2}(\mathbf{r})\right)}\left(\frac{\partial S}{\partial x}\right)^{2}=\left(\omega-v_{\mathrm{p}} \frac{\partial S}{\partial z}\right)^{2}$,

while the trajectory of the wave packet starting at the initial point $x_{3}^{+}, z_{0}$ is given by

$\frac{d z}{V_{\mathrm{g}, \mathrm{z}}}=\frac{d x}{V_{\mathrm{g}, \mathrm{x}}}$,

where $V_{\mathrm{g}, \mathrm{z}}$ and $V_{\mathrm{g}, \mathrm{x}}$ are the components of the group velocities

$$
\begin{aligned}
& V_{\mathrm{g}, \mathrm{z}}=\frac{\partial \omega}{\partial k_{\mathrm{z}}}=v_{\mathrm{p}}-v_{\mathrm{S}}\left(1+\frac{k_{\mathrm{x}}^{2}}{2 k_{\mathrm{z}}^{2}} \frac{v_{\mathrm{S}}^{2}}{v_{\mathrm{S}}^{2}-c_{\mathrm{A}}^{2}}\right) \\
& V_{\mathrm{g}, \mathrm{x}}=\frac{\partial \omega}{\partial k_{\mathrm{x}}}=-\frac{k_{\mathrm{x}}}{k_{\mathrm{z}}} \frac{v_{\mathrm{S}}^{3}}{v_{\mathrm{S}}^{2}-c_{\mathrm{A}}^{2}}
\end{aligned}
$$

If we know how the velocities $v_{S}(\mathbf{r}), v_{\mathrm{p}}(\mathbf{r})$ and $c_{\mathrm{A}}(\mathbf{r})$ depend on coordinates, the system of Eqs. (42)-(45) can be solved numerically. Such a solution allows us to find how the excited wave propagates in the magnetosheath.
We see from the first Eq. (45) that the excited FM wave in the motionless frame is involved in two types of motion: It is convected along the $\mathrm{z}$-axis with the flow velocity $v_{\mathrm{p}}(x)$ and at the same time it moves in the opposite direction with the velocity which is close to the sound speed $v_{\mathrm{S}}$ (neglecting small corrections $\propto k_{\mathrm{x}}^{2} / k_{\mathrm{z}}^{2}$ ). So, the resulting speed along the $\mathrm{z}$-axis is smaller than the flow speed $v_{\mathrm{p}}$. Simultaneously, the wave packet moves along the $\mathrm{x}$-axis with a group velocity given by the second Eq. (45). The wave propagates into the magnetosheath if the corresponding $k_{\mathrm{x}}$ number is negative $k_{\mathrm{x}}<0$ and the relation (43) is satisfied.

It is known that the wave energy $E$ in a moving plasma depends on the reference frame (see e.g McKenzie, 1970):

$E=E_{\mathrm{d}} \frac{\omega}{\omega_{\mathrm{d}}}$,

where $E$ and $E_{\mathrm{d}}$ are the energies in the stationary (magnetospheric) and moving with plasma reference frames. The energy of the FM wave $E_{\mathrm{d}}$ and the frequency $\omega$ are positive while the frequency $\omega_{\mathrm{d}}$ is negative. This means that the energy $E$ in the stationary frame is negative. The x-component of the Poynting flux $\mathbf{P}$

$\mathbf{P}=E \boldsymbol{v}_{\mathrm{g}}$

for the outgoing wave $V_{\mathrm{g}, \mathrm{x}}>0$ is directed from the magnetosheath to the magnetopause. Due to this the FM wave propagating into the magnetosheath raises the energy (the growth rate) of the K.H. instability.

A similar situation takes place for the KA wave propagating into the magnetosheath. As the energy of the KA wave in the frame moving with the plasma is positive, its energy in the stationary (magnetospheric) frame for large flow velocities is negative. The $\mathrm{x}$-component of the Poynting vector is directed towards the magnetopause while the wave propagates into the magnetosheath. In this case, the excited KA wave propagating into the magnetosheath increases the growth rate of instability in the same manner as the FM wave. This problem will be discussed in a separate paper.

\section{Discussion and conclusions}

Low frequency oscillations are commonly detected in the Earth's magnetosheath. Usually it is argued that they can be generated near the bow shock or at the magnetopause. Several different mechanisms of generation of such oscillations are discussed in the literature. In the case of a quasiparallel bow shock, rather strong oscillations convected from the solar wind are observed in the magnetosheath (Lin et al., 1991; Omidi et al., 1994). At the same time it was confirmed by Freeman and Southwood (1988) that the bow shock is transparent only for very low frequency perturbations $\leq 10 \mathrm{mHz}$. For quasi-perpendicular shocks a significant temperature anisotropy $T_{\perp}>T_{\|}$is predicted at the bow shock (Omidi et al., 1994; Krauss-Varban et al., 1994). This 
anisotropy according to the theoretical investigations causes the excitation of the mirror mode and the Alfvén ion cyclotron (AIC) waves (McKean et al., 1992). Being generated at the bow shock, such waves are convected by the solar wind flow into the inner part of the magnetosheath. But the magnetopause is also the source of LF waves. Due to the shear flow, the K.-H. instability can be excited which leads to magnetosonic perturbations. In addition, at the dayside magnetopause a temperature anisotropy $T_{\perp}>T_{\|}$is expected due to the magnetic field draping (Anderson and Fuselier, 1993). This can also result in the excitation of the mirror mode and AIC waves. It should be mentioned that the SM waves are often detected in the magnetosheath. At the same time, there are only a few observations of FM waves being present at the magnetopause and in the magnetosheath (Nagy et al., 2004; Song et al., 1994). One reason for this may be that these waves occur mainly in the higher frequency range of the LF spectrum, which is not so well investigated.

Somewhat similar LF oscillations are observed in the magnetosheath of Mars and Venus (Winningham et al., 2005; Gunell et al., 2008; Espley et al., 2005; Wolff et al., 1980; Biernat et al., 1993; Vörös et al., 2008; Nagy et al., 2004). But the identification of such waves is a rather complicated task due to the lack of detailed information. For example, there is no possibility to receive data from two satellites simultaneously, like for ISEE 1 and 2, or Cluster to detect the direction of the waves propagation, their phase speed and so on. Even the polarization of waves in the magnetosheath of Mars and Venus is not investigated in detail. That is why the theoretical analysis that could provide additional information for comparison with the experimental data is rather important.

A mechanism of LF wave excitation in the planetary magnetosheath associated with the K.-H. instability was suggested in our paper. We argued that in a high $\beta$ magnetosheath plasma with the flow velocity significantly exceeding the sound speed the K.-H. instability causes several types of perturbations simultaneously. First, the SM structure localized in the region of generation should appear. It takes the form of the perturbation attenuated towards the magnetosheath and moving with the averaged flow velocity in the direction of the tail. According to our analysis, this SM mode excites the KA wave in the region where the Doppler-shifted frequency of perturbation becomes equal to the Alfvén frequency. The latter propagates into the magnetosheath at a small angle with respect to the non-disturbed magnetic field.

Another type of oscillation, namely FM waves, could also be detected in a supersonic magnetosheath flow due to the excitation of the K.-H. instability. The FM waves, in general, have a right-handed polarization. In contrast to the SM waves, plasma perturbations associated with the FM waves in the geometric optics approximation are in phase with the perturbations of the longitudinal component of magnetic field, see Eq. (9). Indeed, if the background plasma density and the magnetic field change much more slowly along the $\mathrm{x}$ - axis than the magnetic potential $A_{\mathrm{y}}$, we may differentiate in Eq. (9) only $A_{\mathrm{y}}$. As a result, we find that if $\left|\omega_{\mathrm{d}}\right|$ exceeds the local value $k_{\mathrm{z}} v_{\mathrm{S}}$ along the trajectory, the perturbations of plasma and the longitudinal magnetic field $b_{\|}=\partial A_{\mathrm{y}} / \partial x$ are in phase. But if the background plasma density or the magnetic field also vary rather quickly with $x$, a finite phase shift between the perturbations of plasma and the longitudinal magnetic field appears. So, in real situations these two types of perturbations should not be exactly in phase. The FM waves in high $\beta$ plasma are strongly attenuated due to resonance absorption by ions while propagating along the background magnetic field. At the same time the attenuation of these waves propagating at rather large angles to the magnetic field is small enough. This means that these waves probably could be detected in the inner part of the magnetosheath. The detection of the FM waves in the magnetosheath in high $\beta$ supersonic flow of plasma could be considered as an important confirmation of the excitation of the K.-H. instability near the magnetopause.

\section{Appendix A}

The required solution of Eq. (11) in the vicinity of the third particular point $x_{3}^{+}$can be obtained analytically. Taking into account that in the vicinity of this point $\omega_{\mathrm{d}} \approx-k_{\mathrm{z}} c_{\mathrm{A}}(\sqrt{\beta}+$ $\frac{x-x_{3}^{+}}{L_{v}}$ ) we receive the following approximate equation

$\frac{\partial}{\partial \xi} \frac{1}{\left(\xi-\xi_{3}\right)} \frac{\partial A_{\mathrm{y}}}{\partial \xi}+l^{2} A_{\mathrm{y}}=0$,

where $\xi-\xi_{3}=\frac{x-x_{3}^{+}}{L_{v}}$ and

$l^{2} \approx \frac{\beta-1}{\beta^{3 / 2}} \frac{8 k_{\mathrm{z}}^{2} d^{2} c_{\mathrm{A}}^{2}}{V_{0}^{2}}$

If we introduce a new variable $\eta / l=\left(\xi-\xi_{3}\right)^{3 / 2}$, Eq. (A1) takes the form

$\frac{\partial^{2} A_{\mathrm{y}}}{\partial \eta^{2}}-\frac{1}{3 \eta} \frac{\partial A_{\mathrm{y}}}{\partial \eta}+\frac{4}{9} A_{\mathrm{y}}=0$

This equation has an exact solution in terms of cylindric functions (Kamke, 1959).

$A_{\mathrm{y}}=\eta^{2 / 3} Z_{2 / 3}\left(\frac{2}{3} \eta\right)$

where $Z_{2 / 3}\left(\frac{2}{3} \eta\right)$ is a cylindric function of the order $2 / 3$. We are interested in a solution that corresponds to the propagating wave at $\eta>0$. This solution can be expressed by a combination of Hankel functions. Explicitly our solution for $x>x_{3}^{+}$in general takes the form

$$
\begin{aligned}
A_{\mathrm{y}}= & \left(\xi-\xi_{3}\right)\left[C_{5} H_{2 / 3}^{(1)}\left(\frac{2}{3} l\left(\xi-\xi_{3}\right)^{3 / 2}\right)\right. \\
& \left.+C_{6} H_{2 / 3}^{(2)}\left(\frac{2}{3} l\left(\xi-\xi_{3}\right)^{3 / 2}\right)\right]
\end{aligned}
$$


Here $C_{5}$ and $C_{6}$ are constant quantities that are determined by matching solutions at the boundary between the magnetosheath and the magnetopause. Two terms in the solution (A5) correspond to the waves propagating into the magnetosheath and from the magnetosheath into the magnetopause. Note that despite the resonance at $\xi=\xi_{3}$ in Eq. (A1), the solution of this equation is a regular function of $\xi$ in the vicinity of $\xi=\xi_{3}$.

Now we need to discuss the distribution of the potential in two regions $x>x_{3}^{+}$and $x_{2}^{+}<x<x_{3}^{+}$in more detail. We are interested in the case when the FM wave excited at the point where $\omega_{\mathrm{d}}=-k_{\mathrm{z}} v_{S}$ takes the form of the wave propagating into the magnetosheath. Such a wave near this point is described by the Hankel function of the first type, see solution (A5). This Hankel function can be expressed as a combination of Bessel functions

$H_{2 / 3}^{(1)}(\xi)=\frac{1}{i \sin \left(\frac{2 \pi}{3}\right)}\left[J_{-2 / 3}(\xi)-J_{2 / 3}(\xi) \exp \left(-i \frac{2 \pi}{3}\right)\right](\mathrm{A} 6)$

Taking into account that the Bessel function for small values of its argument is

$J_{v}(\xi)=\left(\frac{\xi}{2}\right)^{v}\left[\frac{1}{\Gamma(v+1)}-\frac{\xi^{2}}{4 \Gamma(v+2)}\right]$

we are in a position to match the solutions in the regions $x>x_{3}^{+}$and $x<x_{3}^{+}$. The required solution describing the outgoing wave for small positive values of argument follows from Eqs. (A6), (A7)

$$
\begin{aligned}
A_{\mathrm{y}}= & -C_{5} \frac{i}{\sin (2 \pi / 3)}\left[\left(\xi-\xi_{3}\right)^{2} \frac{1}{\Gamma(1 / 3)}\left(\frac{l}{3}\right)^{-2 / 3}\right. \\
& \left.-\frac{\exp (-i 2 \pi / 3)}{\Gamma(5 / 3)}\left(\frac{l}{3}\right)^{2 / 3}\right]
\end{aligned}
$$

After the substitution of the solution (A8) into Eq. (22), we find the input of the third term in case of the outgoing FM wave

$$
\begin{aligned}
& -\left.\frac{(\beta+1) f_{\mathrm{d}}^{2}-\beta}{f_{\mathrm{d}}^{2}-\beta} A_{\mathrm{y}}^{*} \frac{\partial A_{\mathrm{y}}}{\partial \rho}\right|_{\rho_{\max }} \\
& \approx 0.55 \frac{\beta^{3 / 2}}{k_{\mathrm{z}} L_{v}}\left|C_{5}\right|^{2} \exp \left(-i \frac{2 \pi}{3}\right)
\end{aligned}
$$

The solution obtained above is valid in the vicinity of the point $x=x_{3}^{+}$. But to find the integrals in Eq. (22) an approximate solution in the whole region $x_{2}^{+}<x<x_{3}^{+}$is required. This solution should coincide with the exact solution found above in the vicinity of the point $x=x_{3}^{+}$. The solution corresponding to the outgoing wave valid for $\xi-\xi_{3}>l^{-2 / 3}$ in an explicit form is the following:

$$
\begin{aligned}
A_{\mathrm{y}} \approx & C_{5}\left(\xi-\xi_{3}\right)^{1 / 4} \frac{(\beta)^{1 / 8}}{2^{1 / 8}\left(k_{\mathrm{z}} L_{v}\right)^{1 / 2}} \\
& \exp \left[i\left(\frac{2^{3 / 2}}{3} \frac{k_{\mathrm{z}} L_{v}}{\beta^{1 / 4}}\left(\xi-\xi_{3}\right)^{3 / 2}-\frac{7 \pi}{12}\right)\right]
\end{aligned}
$$

To find an approximate solution in the region $x_{2}^{+}<x<x_{3}^{+}$, we slightly simplify Eq. (11) assuming $\beta \gg 1$. This new equation we express in the form which is convenient to find a solution in the geometric optics approximation

$$
\frac{\partial}{\partial x} \frac{k_{\mathrm{z}}^{2} c_{\mathrm{A}}^{2}}{\omega_{\mathrm{d}}^{2}-\beta k_{\mathrm{z}}^{2} c_{\mathrm{A}}^{2}} \frac{\partial A_{\mathrm{y}}}{\partial x}+\frac{k_{\mathrm{z}}^{2}}{1+\beta} A_{\mathrm{y}}=0
$$

Let us introduce a new function $A_{1}$ in such a way that

$$
A_{\mathrm{y}}=\left(\frac{\omega_{\mathrm{d}}^{2}}{\beta k_{\mathrm{z}}^{2} c_{\mathrm{A}}^{2}}-1\right)^{1 / 2} A_{1}
$$

After some calculations, we find a solution of Eq. (A11) that corresponds to the outgoing wave for $x>x_{3}^{+}$:

$$
\begin{aligned}
A_{\mathrm{y}}= & C_{3}\left(\frac{\omega_{\mathrm{d}}^{2}}{\beta k_{\mathrm{z}}^{2} c_{\mathrm{A}}^{2}}-1\right)^{1 / 4} \\
& \exp \left[i\left(k_{\mathrm{z}} \int_{x_{3}^{+}}^{x} \sqrt{\frac{\omega_{\mathrm{d}}^{2}}{\beta k_{\mathrm{z}}^{2} c_{\mathrm{A}}^{2}}-1} d x-\frac{\pi}{4}\right)\right]
\end{aligned}
$$

In the vicinity of $x=x_{3}^{+}$solution (A13) takes the form

$$
\begin{aligned}
A_{\mathrm{y}}= & C_{3} \frac{2^{1 / 4}}{\beta^{1 / 8}}\left(\xi-\xi_{3}\right)^{1 / 4} \\
& \exp \left[i \frac{2^{3 / 2}}{3} \frac{k_{\mathrm{z}} L_{v}}{\beta_{s}^{1 / 4}}\left(\xi-\xi_{3}\right)^{3 / 2}-i \frac{\pi}{4}\right]
\end{aligned}
$$

Comparing two solutions (A8) and (A14), we find that

$C_{5}=C_{3}\left(k_{\mathrm{z}} L_{v}\right)^{1 / 2} \frac{2^{3 / 8}}{\beta^{1 / 4}} \exp \left(-i \frac{\pi}{3}\right)$

Note that different phase factors $\exp (-i 7 \pi / 12)$ and $\exp (-i \pi / 4)$ appear in Eqs. (A10) and (A15) because the geometric optics approximation does not give a correct phase factor. The solution in the region $x_{2}^{+}<x<x_{3}^{+}$that corresponds to the solution (A13) in the region $x>x_{3}^{+}$is the following:

$$
\begin{aligned}
A_{\mathrm{y}}= & C_{3}\left(1-\frac{\omega_{\mathrm{d}}^{2}}{\beta k_{\mathrm{z}}^{2} c_{\mathrm{A}}^{2}}\right)^{1 / 4} \\
& {\left[-i \exp \left(\frac{P(x)}{2}\right)+0.5 \exp \left(-\frac{P(x)}{2}\right)\right], }
\end{aligned}
$$

where

$P(x)=2 k_{\mathrm{z}} \frac{\sqrt{\beta}}{\sqrt{\beta+1}} \int_{x}^{x_{3}^{+}} \sqrt{1-\frac{\omega_{\mathrm{d}}^{2}}{\beta k_{\mathrm{z}}^{2} c_{\mathrm{A}}^{2}}} d x$

Parameter

$$
\begin{aligned}
P & =P\left(x_{2}^{+}\right)=2 k_{\mathrm{z}} \frac{\sqrt{\beta}}{\sqrt{\beta+1}} \int_{x_{2}^{+}}^{x_{3}^{+}} \sqrt{1-\frac{\omega_{\mathrm{d}}^{2}}{\beta k_{\mathrm{z}}^{2} c_{\mathrm{A}}^{2}}} d x \\
& \approx 2 \sqrt{\beta}\left(\frac{\pi}{4}-\frac{1}{\sqrt{\beta}}\right) \frac{c_{\mathrm{A}} 2 d k_{\mathrm{z}}}{V_{0}}
\end{aligned}
$$


determines the wave attenuation in the region $x_{2}^{+}<x<x_{3}^{+}$.

With the help of the analysis presented above, we are able to estimate the input to Eq. (22) of the region $x_{2}^{+}<x<x_{3}^{+}$ and the boundary $x=x_{3}^{+}$. Also the distribution of the potential $A_{\mathrm{y}}$ in the region $x_{1}^{-}<x<x_{1}^{+}$is required. An approximate solution in the region $x_{1}^{-}<x<x_{1}^{+}$takes the form

$$
A_{\mathrm{y}}=\left[C_{0}^{(1)} \exp \left(-k_{\mathrm{z}} \sqrt{\frac{\beta}{\beta+1}} x\right)+C_{0}^{(2)} \exp \left(k_{\mathrm{z}} \sqrt{\frac{\beta}{\beta+1}} x\right)\right],
$$

where the coefficients $C_{0}^{(1)}$ and $C_{0}^{(2)}$ are to be determined by matching the solutions of equations for the potential $A_{\mathrm{y}}$ at the boundaries $x_{1}^{+}$and $x_{1}^{-}$. This procedure results in the analysis of complicated equations. Indeed, if $x \rightarrow x_{1}^{+}$, or $x \rightarrow x_{1}^{-}$ the coefficient before the second derivative in Eq. (11) tends towards zero and an equation of the fourth order that takes into account the finite Larmor radius of ions or small dissipation must be analysed. The solutions of such an equation remain finite at the particular points mentioned and approximate matching of solutions of the second order equation near these points can be obtained. This procedure is well known and can be found elsewhere (see, e.g., Taroyan and Erdélyi, 2002 , where the results and the corresponding references are given). In our case when $\beta$, at the outer part of the magnetopause, is supposed to be significantly larger than unity two particular points $x_{1}^{+}$and $x_{2}^{+}$where $(\beta+1) \omega_{\mathrm{d}}^{2}=\beta k_{\mathrm{z}}^{2} c_{\mathrm{A}}^{2}$ and $\omega_{\mathrm{d}}^{2}=k_{\mathrm{Z}}^{2} c_{\mathrm{A}}^{2}$ are close to each other and they influence the result of matching the solutions. This makes the analysis even more complicated. For a rather crude estimate, we consider the case when two particular points $x_{1}^{+}$and $x_{2}^{+}$coincide. Inside the interval $x_{1}^{-}<x<x_{1}^{+}$(except for small regions close to particular points $x_{1}^{+}$and $x_{1}^{-}$) in the case of a symmetric plasmapause the potential $A_{\mathrm{y}}$ approximately takes the form

$$
A_{\mathrm{y}} \approx 0.5 C_{0}\left[\exp \left(-k_{\mathrm{z}} x\right)+\exp \left(k_{\mathrm{z}} x\right)\right]
$$

It can be shown that for high flow velocities $V_{0}$, the jump in the solutions $A_{\mathrm{y}}$ at the particular points $x_{1}^{+}$and $x_{1}^{-}$is small enough (inversely proportional to $V_{0}$ ). As a result the connection between solutions for $x<x_{1}^{+}$and $x>x_{2}^{+}$provides the following result:

$\left|C_{0}\right| \approx \frac{2 \exp (0.5 P)}{\exp \left(-k_{\mathrm{z}} x_{2}^{+}\right)+\exp \left(k_{\mathrm{z}} x_{2}^{+}\right)}\left|C_{3}\right|$

Acknowledgements. The authors would like to thank Max-PlanckSociety for supporting this work by providing travel support. This work also was supported by grant 50QM0801 of the German Aerospace Agency (DLR) and grant MO539/17-1 of the German Science Foundation (DFG).

Edited by: J. Büchner

Reviewed by: two anonymous referees

\section{References}

Anderson, B. J. and Fuselier, S. A.: Magnetic pulsations from $0.1 \mathrm{~Hz}$ to $4.0 \mathrm{~Hz}$ and associated plasma properties in the Earth's subsolar magnetosheath and plasma depletion layer, J. Geophys. Res., 98, 1461-1479, 1993.

Biernat, H. K., Erkaev, N. V., Amerstofer, U. V., Penz, T., and Lichtenegger, H. I. M.: Solar wind flow past Venus and its applications for the occurrence of the Kelvin-Helmholtz instability, Planet. Space Sci., 55, 1793-1803, 2007.

Chandrasekhar, S.: Hydrodynamic and Hydromagnetic Stability, Oxford University Press, Oxford, 1961.

Choudhury, S. R. and Lovelace, R. V. E.: On the Kelvin-Helmholtz instabilities of supersonic shear flow, Astrophys. J., 283, 331342,1984

Dobrowolny, M.: Kelvin-Helmholtz instability in high $\beta$ collisionless plasma, Phys. Fluids, 15, 2263-2270, 1972.

Dobrowolny, M.: Velocity shear instability of Alfvén waves in a high $\beta$ collisionless plasma, Phys. Fluids, 20, 1027-1028, 1977.

Espley, J. R., Cloutier, P. A., Crider, D. H., Brain, D. A., and Akuña, M. H.: Low-frequency plasma oscillations at Mars during the October solar storm, J. Geophys. Res., 110, 25, doi:10.1029/2004JA010935, 2005.

Freeman, M. P. and Southwood, D. J.: The correlation of variations in the IMF with magnetosheath field variations, Adv. Space Res., 8, 217-220, 1988 .

Fujimoto, M., Nakamura, T. K. M., and Hasegawa, T.: Cross-scale coupling within rolled-up MHD-scale vortices and its effect on large-scale plasma mixing across the magnetospheric boundary, Space Sci. Rev., 122, 3-18, 2006.

Fujita, S., Glassmeier, K.-H., and Kamide, K.: MHD waves generated by the Kelvin-Helmholtz instability in a nonuniform magnetosphere, J. Geophys. Res., 101, 27317-27325, 1996.

Glassmeier, K.-H.: ULF pulsations, in: Handbook of Atmospheric Electrodynamics, part 2, edited by: Volland, H., CRC Press, Boca raton. Fla., pp. 463-502, 1995.

Gunell, H., Amerstorfer, U. V., Nilson, H., Grima, C., Koepke, M., Fränz, M., Winnigham, J. D., Frahm, R.A., Sauvaud, J.-A., Fedorov, A., Erkaev, N. V., Biernat, H. K., Holmström, M., Lundin, R., and Barabash, S.: Shear driven waves in the induced magnetosphere of Mars, Plasma Phys. Controlled Fusion, 50, 074018, doi:10.1088/0741-3335/50/7/074018, 2008.

Hasegawa, A. and Chen, L.: Kinetic processes in plasma heating by resonant mode conversion of Alfvén wave, Phys. Fluids, 19, 1924-1934, 1976.

Hasegawa, H., Fujimoto, M., Phan, T.-D., Réme, H., Balogh, A., Dunlop, M. W., Hashimoto, C., and TanDokoro, R.: Transport of solar wind into Earth's magnetosphere through rolled up KelvinHelmholtz vortices, Nature, 430, 755-758, 2004a.

Hasegawa, H., Fujimoto, M., Saito, Y., and Mukai, T: Dense and stagnant ions in the low-latitude boundary region under northward interplanetary magnetic field, Geophys. Res. Lett., 31, L06802, doi:10.1029/2003GL019120, 2004b.

Hasegawa, T., Fujimoto, M., Takagi, K., Saito, Y., Mukai, T., and Réme, H.: Single-spacecraft detection of rolled up KelvinHelmholtz vortices at the flank magnetosphere, J. Geophys. Res., 111, A09203, doi:10.1029/2006JA011728, 2006.

Hasegawa, T., Retinó, A., Vainads, A., Khotyaintsev, Y., André, M., Nakamura, T. K. M., Teh., W.-L., Sonnerup, B. U. Ö., Schwartz, S. T., Seki, Y., Fujimoto, M., Saito, Y., Réme, H., and Canu, P.: 
Kelvin-Helholtz waves at the Earth's magnetopause: Multiscale development and associated reconnection, J. Geophys. Res., 114, A12207, doi:10.1029/2009JA014042, 2009.

Kamke, E.: Differentialgleichungen. Lösungsmethoden und Lösungen. I. Gewönliche Differentialgleichungen, 6th edt., Akad. Verlagsanstalt, Leipzig, 1959.

Krauss-Varban, D.: Bow shock and magnetosheath simulations: Wave transport and kinetic properties, Solar Wind Sources of magnetospheric Ultra-low Frequency waves, AGU, pp. 121-134, 1994.

Lai, S. H. and Lyu, L. H.: Nonlinear evolution of the KelvinHelmholtz instability in a compressible plasma, J. Geophys. Res., 111, AO12202, doi:10.1029/2004JA010724, 2006.

Lin, N., Engebretson, M. J., McPherron, R. J., Kivelson, M. G., Baumjohann, W., Luehr, M., Poterma, T. A., Anderson, B. J., and Zanetti, L. J.: A comparison of ULF fluctuations in the solar wind, magnetosheath, and the dayside magnetosphere. 2. Field and plasma conditions in the magnetosheath, J. Geophys. Res., 96, 3455-3464, 1991.

Mann, I. R., Wright, A. N., Mills, K. J., and Nakaryakov, V. M.: Excitation of magnetospheric waveguide modes by magnetosheath flows, J. Geophys. Res., 104, 333-353, 1999.

McKean, M. E., Winske, D., and Gary, P.: Mirror and ion cyclotron instabilities in the magnetosheath, J. Geophys. Res., 97, 1942119432, 1992.

McKenzie, J. F.: Hydromagnetic wave interaction with the magnetopause and the bow shock, Planet. Space Sci., 18, 1-23, 1970.

Mikhailovskii, A. B. and Onishchenko, O. G.: Nonlinear theory of kinetic Alfvén waves in plasma of high pressure, Phys. Plasma Reports, 21, 357-363, 1995.

Miura, A.: Kelvin-Helmholtz instability at the magnetospheric boundary: dependence $\mathrm{n}$ the magnetosheath sonic Mach number, J. Geophys. Res., 97, 10655-10675, 1992.

Miura, A. and Prichett, P. L.: Nonlocal stability analysis of the MHD Kelvin-Helmholtz instability in a compressible plasma, J. Geophys. Res., 87, 7431-7444, 1982.

Nagy, A. F., Winterhalter, D., Sauer, K., Cravens, T. E., Brecht, S., Mazelle, C., Crider, D., Kallio, E., Zakharov, A., Dubinin, E., Verigin, M., Kotova, G., Axford, W. I., Bertucci, C., and Trotignon, J. G.: The plasma environment of Mars, Space Sci. Rev., 111, 33-114, 2004.

Ohsawa, Y., Nosaki, K., and Hasegawa, A.: Kinetic theory of the hydrodynamic Kelvin-Helmholtz instability, Phys. Fluids, 8, 1139-1143, 1976.
Omidi, N., Farrel, A. O., and Krauss-Varban, D.: Sources of magnetosheath waves and turbulence, Adv. Space Res., 14, 45-54, 1994.

$\mathrm{Pu}, \mathrm{Zu}$-yin and Kivelson, M. G.: Kelvin-Helmholtz instability at the magnetopause: solutions for compressible plasmas, J. Geophys. Res., 88, 841-852, 1983.

Sarafopoulos, D. V., Takahashi, K., and McEntire, R. W.: Periodic variations of magnetosheath energetic electron flux associated with global Pc5 pulsations, J. Geophys. Res., 106, 13037-13052, 2001.

Song, P., Russel, C. T., and Gary, S. P.: Identification of low frequency fluctuations in the terrestrial magnetosheath, J. Geophys. Res., 99, 6011-6025, 1994.

Southwood, D. J.: The hydromagnetic stability of the magnetospheric boundary, Planet. Space Sci., 16, 587-605, 1968.

Takagi, K., Hashimoto, C., Hasegawa, H., Fujimoto, M., and TanDokoro, R.: Kelvin-Helmholtz instability in a magnetotail flanklike geometry: Three dimentional MHD simulations, J. Geophys. Res., 111, A0802, doi:10.1029/2006JA011631, 2006.

Taroyan, Y. and Erdélyi, R.: Resonant and Kelvin-Helmholtz instabilities on the magnetopause, Phys. Plasmas, 9, 3121-3129, 2002.

Vörös, Z., Zwang, T. L., Leubner M. P., Volwerk, M., Delva, M., Baumjohann, W., and Kudela, K.: Magnetic fluctuations and turbulence in the Venus magnetosheath and wake, Geophys. Res. Lett., 35, L11102, doi:10.1029/2008GL033879, 2008.

Winningham, D. J., Frahm, R. A., Shraber, J. R., Coates, A. J., Linder, D. R., Soobiah, Y., Kallio, E., Espley, J. R., Lundin, R., Barabash, S., Holmström, M., Andersson, H., Yamauchi, M., Grigoriev, A., Scherrer, J. R., Jeffers, S. J., Kataria, D. O., Kozyra, J. U., Luhmann, J. G., Roelof, E. C., Williams, D. J., Livi, S., Curtis, C. C., Hsieh, K. C., Sandel, B. R., Koskinen, H., Säles, T., Riihelä, P., Scmidt, W., Grande, M., Carter, M., Sauvaud, J.-A., Fedorov, A., Thocaven, J.-J., McKenna-Lawler, S, Orsini, S., Cerulli-Irelli, R., Maggi, M., Wurz, P., Bochsler, P., Krupp, N., Woch, J., Fränz, M.,.Asamura, K., and Dierker, C.: Electron oscillations in the induced martian magnetosphere, Icarus, 182, 360-370, 2005.

Wolff, R. S., Goldstein, B. E., and Yeates, C. M.: The onset and development of Kelvin-Helmholtz instability at the Venus ionopause, J. Geophys. Res., 85, 7697-7707, 1980. 